Cinémas

Revue d'études cinématographiques

Journal of Film Studies

\title{
Nouvelles technologies et troisième dimension
}

\section{Michel Larouche}

Volume 1, numéro 3, printemps 1991

Nouvelles technologies : nouveaux cinémas?

URI : https://id.erudit.org/iderudit/1001067ar

DOI : https://doi.org/10.7202/1001067ar

Aller au sommaire du numéro

Éditeur(s)

Cinémas

ISSN

1181-6945 (imprimé)

1705-6500 (numérique)

Découvrir la revue

Citer cet article

Larouche, M. (1991). Nouvelles technologies et troisième dimension. Cinémas, 1(3), 77-87. https://doi.org/10.7202/1001067ar

\section{Résumé de l'article}

Diverses technologies sont abordées dans cet article, notamment la synthèse d'images, la stéréoscopie, les frands formats (Imax et Omnimax), la cinéholographie. Si chacune d'elles constitue un pas en avant en vue de la conquête de la troisième dimension et le réalisme de la représentation, l'article démontre que cette quête du réalisme intégral produit l'effet contraire. Une réflexion est développée autour des notions de réalisme et de perception et sur la réintroduction du spectateur dans l'oeuvre. 


\title{
Nouvelles technologies et troisième dimension
}

\section{Michel Larouche}

\begin{abstract}
RÉSUMÉ
Diverses technologies sont abordées dans cet article, notamment la synthèse d'images, la stéréoscopie, les grands formats (Imax et Omnimax), la cinéholographie. Si chacune d'elles constitue un pas en avant en vue de la conquête de la troisième dimension et le réalisme de la représentation, l'article démontre que cette quête du réalisme intégral produit l'effet contraire. Une réflexion est développée autour des notions de réalisme et de perception et sur la réintroduction du spectateur dans l'œuvre.
\end{abstract}

\begin{abstract}
The discussion focuses on a range of technologies including image synthesis, stereoscopy, large-scale formats (Imax, Omnimax), and cineholography. While each of these technologies apparently constitutes a step towards the conquest of the third dimension and realism in representation, the article shows that, in fact, they have had the opposite effect. The meaning of the notions of realism and perception and the reintroduction of the spectator into the work are considered.
\end{abstract}

Du seul fait qu'il soit composé de photogrammes qui s'apparentent à des photographies, le cinéma offre déjà une certaine tridimensionalité. Comme la photo, il reproduit une représentation codée de la troisième dimension (la perspective) sur une surface plane (deux dimensions). De plus, par le mouvement, les effigies immobiles se voient arrachées à la surface plane: le mouvement apporte le relief. «En somme, le secret du cinéma - dit Metz —, c'est d'arriver à mettre beaucoup 
d'indices de réalité dans des images, qui, ainsi enrichies, restent néanmoins perçues comme images» (p. 23).

À première vue, le développement des nouvelles technologies au cinéma s'effectue sous le signe d'une accentuation des indices de réalité, par l'apport de nouveaux éléments à cette relation images analogiques/mouvement/relief qui constitue la base même du cinéma, autrement dit la tridimensionalité.

Lorsqu'on parle de tridimensionalité, on pense spontanément aux films réalisés en images de synthèse, dits en $3 \mathrm{D}$. Après une période où l'utilisation de l'ordinateur se limitait à une technique d'interpolation pour effectuer les transitions afin de compléter le mouvement entre un dessin de départ exécuté par un artiste sur un terminal graphique et un dessin d'arrivée, on a mis au point la technique de numérisation rendant possible l'«effet volume». La troisième dimension est désormais inscrite dans le programme de l'ordinateur. La synthèse d'images peut alors imiter l'analogique par des procédés de simulation, mais au niveau du résultat présenté à l'écran elle reproduit toujours la représentation codée du cinéma narratif conventionnel. La conception assistée par ordinateur ne se présente pas d'emblée comme une réponse à la volonté de conquête de la troisième dimension. C'est plutôt du coté de l'expanded cinema ${ }^{1}$ qu'il faut aller pour assister à un progrès dans cette direction.

À cet égard le cinéma Imax, étant donné la dimension gigantesque de son écran (dont les extrémités correspondent aux limites de la vision périphérique du spectateur) et sa grande qualité d'image (en ce qui a trait notamment à sa luminosité), engendre un pas en avant dans le développement du relief, intensifiant de la sorte le caractère tridimensionnel des images. Le cinéma Omnimax accroît encore davantage la représentation tridimensionnelle: les images sont enregistrées selon une perspective curviligne (grâce à l'objectif fish eye) et projetées sur un écran semi-hémisphérique: il y a quasi-suppression du cadre et la représentation respecte la vision dans le champ visuel total de l'œil (analogie avec l'image rétinienne) contrairement à la perspective classique.

Le cinéma stéréoscopique, aussi appelé "cinéma en relief", propose aussi une tridimensionalité mais d'un autre ordre: il est le résultat d'une illusion fondée sur le principe du parallaxe binoculaire et qui nécessite le port de lunettes, vieux procédé largement répandu pendant les années 50. La stéréoscopie est présentement ravivée par les cinémas Imax et Omnimax ${ }^{2}$ : le spectateur d'un film stéréoscopique est alors confronté à des images qu'il peut littéralement toucher... 


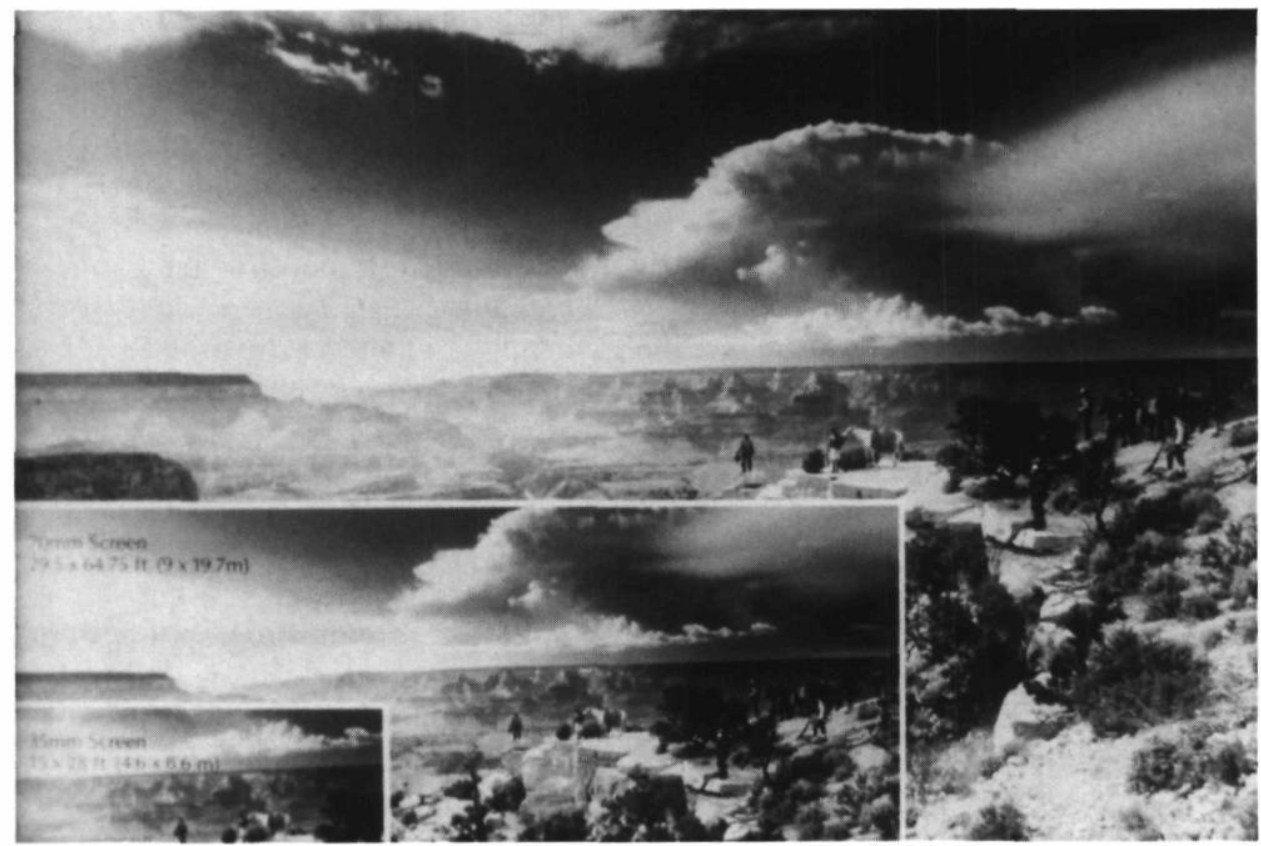

Comparaison entre les formats $35 \mathrm{~mm}, 70 \mathrm{~mm}$ et Imax

\section{0}

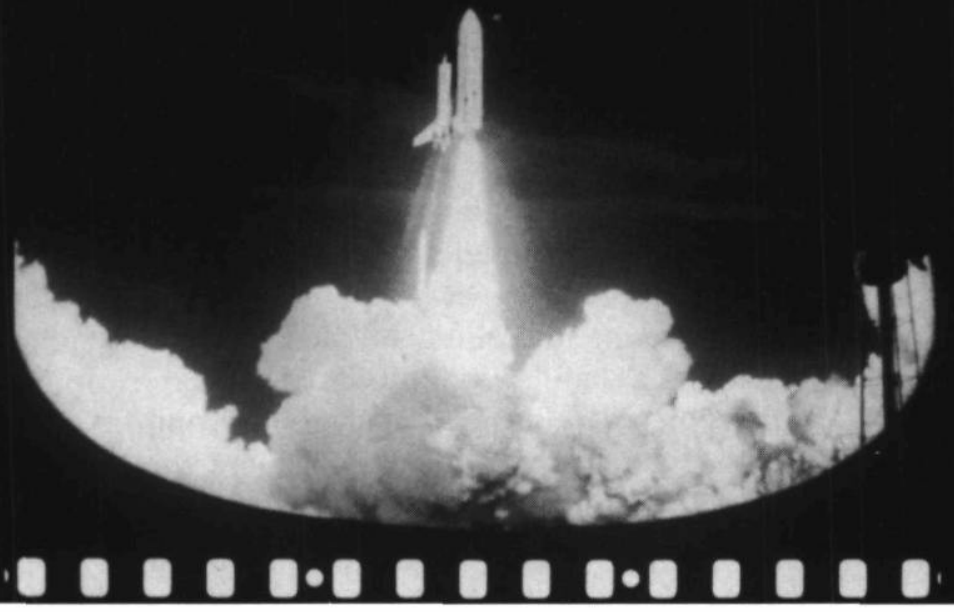

Photogramme du film Omnimax En direct de l'espace de Graeme Ferguson (1985) 
Quant à la cinéholographie, elle apporte le relief intégral. La troisième dimension n'est plus le fruit d'une illusion comme c'est le cas avec la stéréoscopie, elle est réellement présente dans un message sous code optique qui contient des informations complètes concernant le relief, la perspective, la profondeur de champ.

Le programme en vue de la maîtrise de la troisième dimension apparaît donc riche et les prouesses technologiques se succèdent afin de produire le réalisme intégral tant souhaité. On assiste ainsi au premier film Imax stéréoscopique, Transitions (1986), par Colin Low et Tony Ianzelo, après des années de recherche en vue de fabriquer un support spécial pour la caméra stéréoscopique. Avant de réaliser son film, Colin Low a longuement réfléchi sur la stéréoscopie appliquée aux grands formats. Dans un texte intitulé: «Grand écran et relief - Considérations esthétiques et techniques», il parle de l'intérêt du format Imax pour l'espace stéréoscopique: étant donné la quasi-disparition du cadre, non seulement de petits objets mais un personnage peut «sortir» de l'écran pour venir dans la salle. Puis après la mise au point par la société Imax d'un caisson d'insonorisation destiné à atténuer le bruit produit par la caméra en marche, les mêmes cinéastes réalisent Urgence (1988), film novateur par l'importance des dialogues intimistes et qui ouvre la voie au tournage de dramatiques en système Imax. Enfin Le Premier Empereur de Chine (1989), réalisé en Omnimax par Tony Ianzelo et Lio Hao Xue, poursuit l'exploration des possibilités qu'offre le médium dans les films de fiction. Or toutes ces expérimentations qui apportent des indices de réalité supplémentaires ne produisent pas la fameuse impression de réalité du cinéma conventionnel.

En ce qui a trait à la cinéholographie, on assiste à un phénomène semblable. Elle présente un relief intégral perceptible par une vision monoculaire (contrairement à la stéréoscopie qui nécessite une bonne vision binoculaire), et donne des images qui peuvent paraître extrêmement réelles et communiquer ainsi la sensation de la présence de l'objet avec une grande efficacité, mais étant donné que le mode d'enregistrement des images en cinéholographie est lié à l'utilisation du laser, l'image produite sera toujours transparente (elle ne projette pas d'ombre) et immatérielle. La présence intégrale de la troisième dimension ne peut donc servir, en cinéholographie, des visées réalistes.

Il est étonnant de constater que la synthèse d'images s'est vouée elle aussi à l'obtention du réalisme de la représentation et 
la simulation de l'être humain. Philippe Bergeron précise, à propos de Tony de Peltrie (1985):

C'était un premier pas nécessaire. Il comporte bien des défauts. Tony est à la fois gauche et un peu trop parfait. Il n'a pas les imperfections des êtres vivants. Un détail: sa chemise lui colle à la peau... L'une des difficultés majeures de l'animation sur ordinateur est précisément de simuler ces imperfections, ces irrégularités du cheveu ou du vêtement qui sont celles des êtres humains ${ }^{3}$.

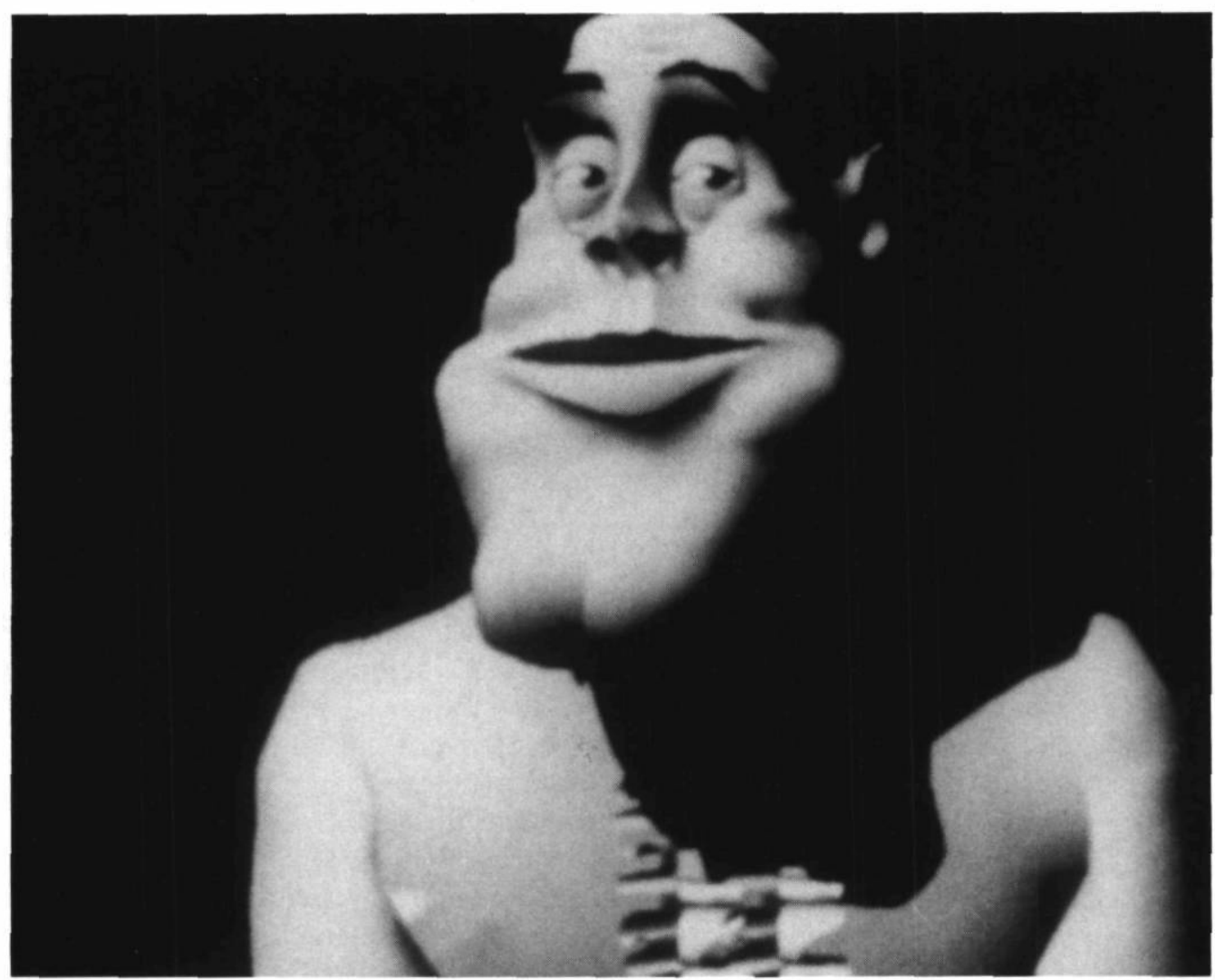

Tony de Peltrie de Philippe Bergeron, Pierre Lachapelle, Daniel Langlois, Pierre Robidoux (1985)

Coll. Cinémathèque québécoise

Lorsqu'il est venu à "Convergence II: au-delà de la technologie», à l'automne 1986 à Montréal, il parlait même très sérieusement de la disparition prochaine des acteurs de cinéma. Nadia Magnenat-Thalmann, après son travail sur Rendez-vous à Montréal (1987, coréalisé avec Daniel Thalmann), en arrivait à la conclusion suivante: 
On est donc capables dorénavant de faire des acteurs électroniques, totalement dépendants d'un modèle dynamique qui bouge. Et alors rien ne nous empêche de réunir dans un même bureau à côté de moi John Kennedy, Pierre Eliott Trudeau et, par exemple, Gorbatchev et puis bon, on pourrait boire le thé ou discuter. Donc on peut faire dire aux gens n'importe quoi, leur faire faire n'importe laquelle expression tout en respectant leur personnalité 4 .

Or dans le cas des images de synthèse comme pour les films Imax, Omnimax et stéréoscopiques, la réussite technologique n'est pas garante de succès artistique. Dans Rendez-vous à Montréal, on a cherché à faire revivre Marilyn Monroe et Humphrey Bogart (mais on ne confond toujours pas les faux acteurs des vrais) et le film constitue un pas en avant dans le développement de l'intelligence artificielle. On comprend toutefois le point de vue de Dominique Noguez qui, invité au 6e «Rendezvous du cinéma québécois» (février 1988), parle de la «nullité des essais à l'ordinateur» (p. 5) alors qu'on y présente Rendezvous à Montréal, qui au même moment, pour son innovation technologique, est illustré sur la page couverture d'un numéro de La Recherche. Rappeler l'exploit technologique en expliquant que tel geste de la main a coûté des mois de travail et des sommes colossales ne change rien aux critères de qualité artistique.

Douglas Kay, qui travaille à l'ILM (la société d'effets spéciaux fondée par George Lucas) précise: «La simulation de la réalité est juste un moyen de tester la qualité des techniques... techniques qui, utilisées tout autrement, permettront d'obtenir des résultats différents» (Cazals, p. 53). Et Florence de Mèredieu ajoute: «Il y aurait effectivement grande naïveté à croire qu'une technique neuve engendre ipso facto des images neuves. Les révolutions artistiques suivent généralement les révolutions techniques avec un décalage» (p. 58). La quête de réalisme ne sert donc bien souvent qu'à reconduire, avec des techniques neuves, une vieille imagerie, sans les conventions et les codes qui fondaient son caractère figuratif. On constate alors plus que jamais que le «code perceptif continue à régir notre idéologie de la représentation, alors même que ses règles sont ignorées du plus grand nombre» (Gauthier, p. 24). Le caractère construit de l'image ne peut plus se faire oublier. Une rupture s'instaure entre indices supplémentaires de réalité et impression de réalité, et partant, entre tridimensionalité et impression de réalité. Autrement dit, un écart se creuse entre le caractère conventionnel de la représentation figurative et le réalisme de la représentation. Et ressort avec acuité le point de vue de Éco, repris et développé par Dominique Chateau (p. 61), selon lequel le signe iconique ne possède pas les propriétés de l'objet représenté mais traduit des propriétés pré- 
analysées de cet objet. La conquête de la tridimensionalité, en accentuant le transfert en images d'attributs des objets du monde visible, permet enfin d'affronter le problème de la nature du réel, qu'Edgar Morin considérait comme la mission du cinéma du réel:

C'est-à-dire que le cinéma qui se pose les plus graves et les plus difficiles problèmes par rapport à l'illusion, l'irréalité, la fiction, est bien le cinéma du réel, dont la mission est d'affronter le plus difficile problème posé par la philosophie depuis deux millénaires: celui de la nature du réel (p. 3).

Les nouvelles technologies obligent à un déplacement majeur: de la nature du réel, on passe non plus seulement à la nature de la représentation mais à la nature de la perception. Le réel semble désormais une affaire de stricte perception. Force est donc de constater que les nouvelles technologies appliquées au cinéma, ou bien n'ont pas encore réussi à maîtriser les conventions du réalisme, ou encore que leur voie se dessine ailleurs, vers de nouveaux cinémas.

Questionnons ces technologies selon leurs caractéristiques «intrinsèques». Jean-Louis Weissberg affirme dans Paysages virtuels:

[Des] techniques telles que l'holographie, ou le cinéma hémisphérique comme à la Géode de la Villette, explorent la représentation tridimensionnelle. Mais passés les premiers moments, l'accoutumance recompose la perception par analogie avec les situations habituelles de face à face. Sans dénier la performance ni l'introduction à une nouvelle forme de spectacle, on demeure dans la re-présentation (p. 26).

Ces propos conviennent aux œuvres réalisées présentement selon une vieille imagerie et expliquent leurs ambiguïtés esthétiques. Mais ils s'appliquent mal aux enjeux esthétiques fondamentaux qu'elles génèrent. En ce qui a trait à la cinéholographie, Guy Fihman parle de «sculptures en mouvement» par rapport au cinéma conventionnel qui serait de la «peinture en mouvement» ${ }^{5}$. Et Philippe Jaulmes, qui a beaucoup réfléchi sur les possibilités des grands formats et plus précisément sur le procédé Panrama devenu l'Omnimax, donne les règles de ce nouveau cinéma, d'une narrativité fondée sur la logique visuelle des spectateurs et non plus sur les conventions articulantes du découpage cinématographique:

Il est une réponse à des critères jugés prioritaires: la restructuration de l'espace et la participation du spectateur. Il n'est pas l'extrapolation en plus grand de ce qui existe déjà, il n'est pas un compromis pour attirer le public, il n'est pas une amélioration technique de plus après la couleur et le cinémascope. Il se veut un 
nouveau cinéma, une nouvelle façon de voir et de communiquer (p. 13-14).

Dans la réalisation des films générés à l'aide de l'ordinateur, la caméra obscura n'est plus le préalable nécessaire à la représentation du monde, l'analyse structurale remplaçant désormais l'observation optique, c'est pourquoi on qualifie ces œuvres d'images de synthèse: la lumière, la couleur, l'angle et le mouvement de caméra viennent par la suite. À partir du moment où on atteint le réalisme, il est alors possible de s'en libérer et la voie s'ouvre à l'imaginaire. Des objets très réalistes peuvent se métamorphoser avec aisance, des solides se transformer en formes molles, produisant un vertige du surréel chez le spectateur, confronté à un monde à la fois palpable et invraisemblable. Une fois l'objet donné en volume à l'ordinateur, il est tellement facile de produire les angles les plus inusités, les mouvements les plus inattendus! L'image n'est plus réelle, elle est virtuelle (un choix parmi une possibilité de $\mathrm{X}$ images) et se présente d'emblée interactive. Il est, de plus, étonnant de constater jusqu'à quel point les images de synthèse s'intègrent fort bien aux films Imax et Omnimax, qui enclenchent spontanément vers la participation du spectateur, de même qu'à la stéréoscopie.

Cette dernière offre en effet un «indice de réalité» de surface. Avec la profondeur de champ, le cinéma conventionnel a déjà un certain relief mais qui reste «en arrière» de l'écran, dans les limites «classiques» de l'espace du film. La stéréoscopie est l'inverse de la profondeur de champ, comme si l'écran devenait projecteur. Le gain de réalité par rapport au cinéma traditionnel brise la séparation «monde imaginaire de la diégèse»/«monde réel de la salle de cinéma». L'espace du film pénètre dans l'espace du spectateur.

Lorsqu'on aborde les nouvelles technologies, il faut sortir de la relation conventionnelle qui a toujours défini le spectacle cinématographique. Le spectateur joue dorénavant un rôle physiquement actif et le spectacle cinématographique devient un lieu de performance. Cette dernière, caractérisée par un excès énergétique favorisant une confusion entre sens et référence, questionne le statut du réel par absence de renvoi à la coïncidence. «Elle nous introduit ainsi à ce que nous pourrions appeler l'espace du dérapage» (Pelzer, p. 29), qui «mime la référence plus qu'il ne l'exécute» (p. 30). «Revendiquant une certaine gratuité et comme un droit à l'anecdote, elle semble vouloir échapper à un héritage déjà lourd fait de formalisme, de rupture et de raréfaction du désir» (p. 31). 
Si les œuvres issues des nouvelles technologies ont opté bien souvent pour l'imitation, la re-présentation, offrant des images simulacres, le mouvement intérieur qui les anime se situe loin de ces concepts. Le passage d'une structure ternaire à une structure duelle semble bien le commun dénominateur des nouvelles technologies. Cette modification dans le statut du spectateur était déjà présente dans les œuvres qualifiées de «nouvelles images», "postmodernes», «films d'effets», de même que dans la vidéo considérée à la fois comme support et esthétique et dont la nature hybride a largement été étudiée. Analysant le Métropolis de Moroder, Odin en arrive à la conclusion suivante:

(...) le film agit directement sur son spectateur, un spectateur qui ne vibre plus tant aux événements racontés (effet fiction) qu'aux variations de rythme, d'intensité et de couleur des images et des sons. Le lieu du film se déplace ainsi de l'histoire vers les vibrations diffusées dans la salle par le complexe plastico-musical agissant en tant que tel. C'est, désormais, ce complexe qui règle le positionnement du spectateur sans passer par la médiation d'un tiers symbolisant. C'est que la communication n'a pas ici pour objet privilégié la production de sens mais la production d'affects (p. 134).

Si les images numériques reproduisent la représentation codée du cinéma conventionnel en regard du produit présenté à l'écran, étant donné la dimension interactive qui se situe au niveau de leur genèse (la programmation) et leur statut virtuel (la possibilité de remodeler l'œuvre à volonté à partir des données informatiques introduites dans l'ordinateur et l'éventuel accès au code de la part du public) elles relèvent d'enjeux semblables à ceux des films Imax et Omnimax, stéréoscopiques et holographiques en ce qui a trait à la participation du spectateur. Cette caractéristique est aussi partagée par les disques laser interactifs (dont les images présentement analogiques s'annoncent numériques) qui proposent une dynamique selon laquelle la relation du spectateur à l'œuvre est, cette fois, immédiate.

Cette avenue qui explore l'introduction du spectateur dans l'œuvre, commun dénominateur des nouvelles technologies, ouvre à une nouvelle tridimensionalité. «La simulation interactive, dit encore Jean-Louis Weissberg, dans la mesure où elle contraint l'interactant à éprouver la profondeur de champ en pénétrant dans la représentation, actualise en revanche la notion de relief» (p. 26). L'inclusion du spectateur dans l'œuvre et la multiplicité des points de vue brise en quelque sorte cette continuité avec la mimésis échafaudée depuis la Renaissance. Si, en apparence, les nouvelles technologies appliquées au cinéma nous ramènent à une esthétique issue du Quattrocento, la désincarnation du visuel, le caractère purement fantomatique des images 
produisent un réalisme abstrait qui nous éloigne totalement de la mimésis. C'est à une pensée préperspectiviste à laquelle nous sommes confrontés, où la vraisemblance visuelle n'organise plus la représentation de l'espace, où le temps n'est plus homogène, continu, durée linéaire.

Le point de vue selon lequel seules les images de synthèse, étant donné leur nature numérique, s'inscrivent en faux contre le modèle perspectiviste est à relativiser. Il y a certes rupture avec l'analogique des images conventionnelles, mais non pas celui des nouvelles images en général. Le mot analogique ne recouvre plus le même champ sémantique qu'auparavant. Une méconnaissance des technologies autres que numériques (films Imax, Omnimax, stéréoscopiques, holographiques, etc.) peut expliquer ce malentendu. Les images de synthèse côtoient sans problème l'analogique, jusqu'à occasionner un effondrement de tous les systèmes d'analyse. Le terme «hybride» est devenu le mot clé pour identifier à la fois la rupture et le mariage. Si les deux vont si bien ensemble, c'est qu'un déplacement perceptuel s'est opéré, et la rupture d'avec le modèle renaissant se situe davantage à ce niveau qu'à celui de la seule iltnovation technologique.

Revenons à notre programme images analogiques/mouvement/relief qui a toujours déterminé le caractère tridimensionnel du cinéma. Nous constatons qu'avec les nouvelles technologies c'est l'expression images analogiques qui pose problème. Un abîme s'est creusé entre représentation figurative et représentation photographique. Surgit alors une troisième dimension dont les enjeux créatifs ouvrent grandes les portes à un univers subjectif aux possibilités inouïes. Reprenons les propos de Metz cités en introduction: «En somme, le secret du cinéma, c'est d'arriver à mettre beaucoup d'indices de réalité dans des images, qui, ainsi enrichies, restent néanmoins perçues comme images» (p. 23). C'est la signification même de l'expression «indices de réalité» qui se modifie radicalement lorsqu'on aborde les nouvelles technologies. Une réflexion sur la troisième dimension, tout en mettant en valeur les particularités de chaque technologie, témoigne d'un changement en profondeur en train de s'opérer, symptôme peut-être de la mort d'un cinéma ou de la naissance de nouveaux cinémas, à moins que l'on parle de cinéma en mutation, d'un déplacement certain en tous cas des données les plus fondamentales au sein de la théorie cinématographique. 


\section{NOTES}

1 Expression utilisée par Gene Youngblood pour qualifier les «débordements» que le développement contemporain des techniques audio-visuelles permet au cinéma. Voir à ce sujet Expanded Cinema (New York: E.P. Dutton \& Co, 1970).

2 La grandeur du format permet de régler certains des problèmes techniques rencontrés par la stéréoscopie.

${ }^{3}$ Propos tirés de Georges Turner, «Electronic characterization», American Cinematographer (juillet 1986), traduits et repris dans «Images de synthèse: un art?», Dossiers de l'audiovisuel 15 (sept.-oct. 1987) p. 47.

4 Propos tirés du vidéo Rendez-vous à Montréal, le film du film, par Nadia Magnenat-Thalmann et Daniel Thalmann (1987).

5 Propos émis lors de la session «Expériences en communication visuelle», lors du colloque Convergence II: au-delà de la technologie, tenu à l'automne 1986 à Montréal.

\section{OUVRAGES CITÉS}

Cazals, Thierry. «Le monde comme simulacre et programmation». Cahiers du cinéma 399 (sept. 1987).

Chateau, Dominique. «Film et réalité: pour rajeunir un vieux problème». Iris vol. 1 no 1 (1983).

Gauthier, Guy. Vingt leçons sur l'image et le sens. Paris: Edilig, 1982.

Jaulmes, Philippe. L'Écran total, pour un cinéma sphérique. Paris: Lherminier, 1981.

Low, Colin. «Grand écran et relief - Considérations esthétiques et techniques». Perforations vol. 3 no 4 (1983).

Mèredieu, Florence de. "L'art — et son double - (l'art)». Paysages virtuels. Paris: Dis Voir, 1988.

Metz, Christian. Essais sur la signification au cinéma. Tome I. Paris: Klincksieck, 1971.

Morin, Edgar. [Présentation]. Cinéma du réel. Festival international du film ethnographique et sociologique. Paris: Centre Georges Pompidou, 1980.

Noguez, Dominique. «Un cinéma de quadragénaires?». Copie zéro 36 (août 1988).

Odin, Roger. «Du spectateur fictionnalisant au nouveau spectateur. Approche sémio-pragmatique». Iris 8 (1988).

Pelzer, Birgit. «La Performance ou l'Intégrale des équivoques». Performance text(e)s \& documents. Montréal: Parachute, 1981.

Weissberg, Jean-Louis. "Sous les vagues la plage». Paysages virtuels. Paris: Dis Voir, 1988. 